\title{
Markers of sulfadoxine-pyrimethamine resistance in Eastern Democratic Republic of Congo; implications for malaria chemoprevention
}

\begin{abstract}
Marit van Lenthe ${ }^{1 * \dagger} \mathbb{0}$, Renske van der Meulen ${ }^{1,2+}$, Maryvonne Lassovski $^{7 \wedge}$, Adelaide Ouabo $^{6}$, Edwige Bakula $^{7}$, Colette Badio ${ }^{7}$, Deogratias Cibenda ${ }^{5}$, Lucy Okell ${ }^{8}$, Erwan Piriou', Lynn Grignard ${ }^{3}$, Kjerstin Lanke ${ }^{2}$, Bhargavi Rao ${ }^{4}$, Teun Bousema ${ }^{2 \dagger}$ and Cally Roper ${ }^{3+}$
\end{abstract}

\begin{abstract}
Background: Sulfadoxine-pyrimethamine (SP) is a cornerstone of malaria chemoprophylaxis and is considered for programmes in the Democratic Republic of Congo (DRC). However, SP efficacy is threatened by drug resistance, that is conferred by mutations in the $d h f r$ and dhps genes. The World Health Organization has specified that intermittent preventive treatment for infants (IPTi) with SP should be implemented only if the prevalence of the dhps K540E mutation is under $50 \%$. There are limited current data on the prevalence of resistance-conferring mutations available from Eastern DRC. The current study aimed to address this knowledge gap.

Methods: Dried blood-spot samples were collected from clinically suspected malaria patients [outpatient department (OPD)] and pregnant women attending antenatal care (ANC) in four sites in North and South Kivu, DRC. Quantitative PCR ( $(\mathrm{PCCR}$ ) was performed on samples from individuals with positive and with negative rapid diagnostic test (RDT) results. Dhps K450E and A581G and dhfr $1164 \mathrm{~L}$ were assessed by nested PCR followed by allele-specific primer extension and detection by multiplex bead-based assays.

Results: Across populations, Plasmodium falciparum parasite prevalence was 47.9\% (1160/2421) by RDT and 71.7 (1763/2421) by qPCR. Median parasite density measured by qPCR in RDT-negative qPCR-positive samples was very low with a median of 2.3 parasites/ $\mu \mathrm{L}$ (IQR 0.5-25.2). Resistance genotyping was successfully performed in RDT-positive samples and RDT-negative/qPCR-positive samples with success rates of 86.2\% (937/1086) and 55.5\% (361/651), respectively. The presence of $d$ hps K540E was high across sites (50.3-87.9\%), with strong evidence for differences between sites ( $p$ 0.001). Dhps A581G mutants were less prevalent $(12.7-47.2 \%)$. The dhfr $1164 \mathrm{~L}$ mutation was found in one sample.
\end{abstract}

Conclusions: The prevalence of the SP resistance marker dhps K540E exceeds 50\% in all four study sites in North and South Kivu, DRC. K540E mutations regularly co-occurred with mutations in dhps A581G but not with the dhfr 1164L mutation. The current results do not support implementation of IPTi with SP in the study area.

\footnotetext{
*Correspondence: marit.van.lenthe@amsterdam.msf.org

Maryvonne Lassovski-deceased

${ }^{\dagger}$ Marit van Lenthe, Renske van der Meulen, Teun Bousema and Cally

Roper contributed equally to this work

${ }^{1}$ Médecins Sans Frontières (MSF), Amsterdam, The Netherlands

Full list of author information is available at the end of the article
}

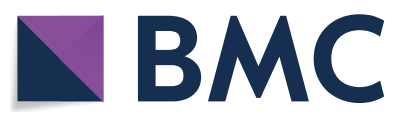

(c) The Author(s) 2019. This article is licensed under a Creative Commons Attribution 4.0 International License, which permits use, sharing, adaptation, distribution and reproduction in any medium or format, as long as you give appropriate credit to the original author(s) and the source, provide a link to the Creative Commons licence, and indicate if changes were made. The images or other third party material in this article are included in the article's Creative Commons licence, unless indicated otherwise in a credit line to the material. If material is not included in the article's Creative Commons licence and your intended use is not permitted by statutory regulation or exceeds the permitted use, you will need to obtain permission directly from the copyright holder. To view a copy of this licence, visit http://creativeco mmons.org/licenses/by/4.0/. The Creative Commons Public Domain Dedication waiver (http://creativecommons.org/publicdomain/ zero/1.0/) applies to the data made available in this article, unless otherwise stated in a credit line to the data. 
Keywords: Malaria, DRC, Sulfadoxine-pyrimethamine (SP), Chemoprophylaxis, dhps, dhfr, K540E, A581G, I164L, IPTi

\section{Background}

Despite a decline in cases and deaths over the past 20 years, malaria forms a major health problem in Africa. In the Democratic Republic of Congo (DRC) cases of malaria are rising, and the death toll is increasing [1]. In 2017 in DRC there was an estimated number of 25 million (15.7-36.8) cases of malaria and an estimated 46,800 $(36,200-57,300)$ deaths. The majority of malaria cases in DRC are caused by Plasmodium falciparum, although Plasmodium ovale [2], Plasmodium malariae, and Plasmodium vivax are present among symptomatic cases [3]. Médecins sans Frontières (MSF) has been working in DRC since 1991. The Operational Centre Amsterdam of MSF (MSF-OCA) has several projects in Eastern DRC, most notably in North and South Kivu. Both provinces are the scene of chronic and at times intense conflict, leading to frequent internal displacement, outbreaks of disease, sexual violence, malnutrition, and a collapse of healthcare services. MSF supports several large hospitals and health centres and has adopted a strategy of Integrated Community Case Management (ICCM). Within the ICCM programme, community members are trained to diagnose and treat malaria, diarrhoea and pneumonia in children under 5 years of age. MSF and the Ministry of Health distribute insecticide-treated bed nets in the community as well as targeted distribution to pregnant women, severe malaria patients and other specific groups. Additional interventions are implemented where supportive evidence warrants it, aiming to reduce the burden of malaria. These interventions include chemoprevention strategies that are delivered without the need for testing. Sulfadoxine-pyrimethamine (SP) is a cornerstone of malaria chemoprophylaxis across the African continent. Intermittent preventive treatment of malaria in pregnancy (IPTp) and intermittent preventive therapy for infants (IPTi) use SP alone, while seasonal malaria chemoprophylaxis (SMC) combines SP with amodiaquine. The efficacy of SP for these interventions is influenced by drug resistance levels in parasite populations [4] and the drug resistance levels are influenced by the use of SP in target populations $[5,6]$.

The genetic basis of SP resistance is well understood, and consequently molecular surveillance of resistance mutations underpins current policy on SP use in Africa. SP resistance occurs via the accumulation of mutations in the dihydrofolate reductase ( $d h f r$ ) gene and in the dihydropteroate synthase (dhps) gene and these progressively reduce susceptibility to pyrimethamine and sulfadoxine, respectively. Infections harbouring triple mutant $d h f r$, i.e., a combination of N51I, C59R and S108N mutations, are common throughout Africa. When this triple mutation in dhfr is combined with double-mutant dhps (A437G and K540E) the risk of SP treatment failure is up to $75 \%[7,8]$. A 'sextuple mutant' genotype in which these five key mutations in $d h f r$ and dhps gene are accompanied by dhps A581G is increasingly reported [9].

The World Health Organization (WHO) has specified that IPTi-SP should only be implemented in areas where the prevalence of the dhps K540E mutation (a proxy measure for the presence of all 5 key mutations) is under $50 \%$ [10]. Molecular surveillance reports up to 2016 have recorded prevalence of K540E exceeding the 50\% threshold in 12 countries: most in East Africa (Sudan, Somalia, the Republic of the Congo, Tanzania, Malawi, Kenya, DRC, Mozambique, Ethiopia, Rwanda, Uganda, Zambia) [9], with relevant within-country variation. In the case of IPTp, SP continues to provide protection in pregnancy in all these areas except within restricted geographic areas where the dhps $581 \mathrm{G}$ is also present at high prevalence $[4,11-14]$. One such area is confined to southwest Uganda, Rwanda and bordering areas of Eastern DRC. A study in 2013-2014 to survey small numbers of samples from all 25 districts of DRC found that prevalence of the sextuple mutant genotype is concentrated in North and South Kivu (Fig. 1), which are the districts bordering southwestern Uganda and Rwanda.

There are indications that the range and prevalence of the sextuple genotype is increasing [9]. This study aimed to update molecular surveillance data from North and South Kivu with more substantial sample sizes and examine the geographic heterogeneity in prevalence of $540 \mathrm{E}$ and $581 \mathrm{G}$ in order to determine if IPTi could be a preventive strategy used in MSF mission sites within these provinces of Eastern DRC.

\section{Methods}

\section{Study area, subjects and sample collection}

From August to September 2017, samples were collected in the context of MSF projects in two provinces: North Kivu and South Kivu. Each province provided samples from two study sites. The aim was to obtain a minimum of 250 samples with confirmed P. falciparum infection per study site from either pregnant women attending ANC or individuals attending the clinic with suspected malaria.

The sample size was based on the assumption of $50 \%$ prevalence of dhps K540E and the ability to estimate this expected proportion with $5 \%$ precision for each site. The 


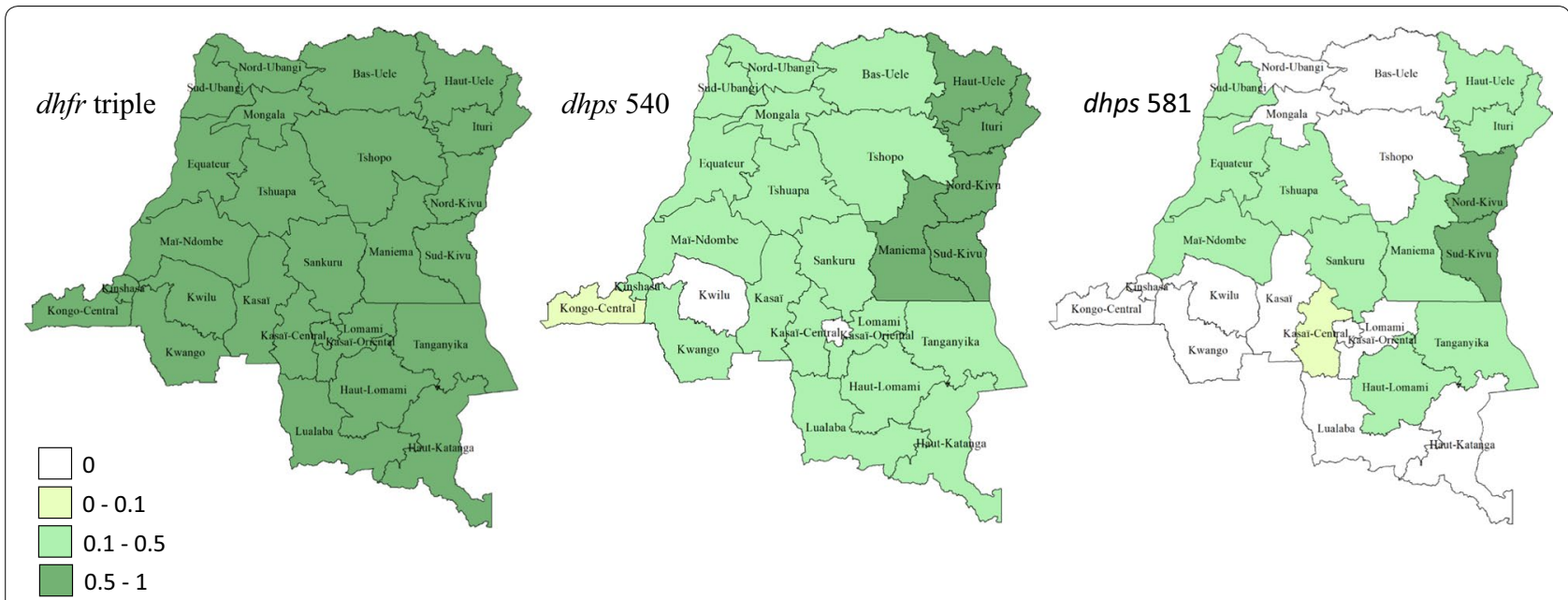

Fig. 1 The prevalence of SP resistance markers. The prevalence of SP resistance markers in each of the 25 districts of the DRC $2013-2014$ (redrawn from [25]). Administrative districts are shaded according to the prevalence of mutations. a The 'dhfr triple' containing N511,C59R and S108N. b The dhps K540E. c The dhps A581G. SP sulphadoxine-pyrimethamine, dhfr dihydrofolate reductase, dhps dihydropteroate synthase

study received ethical approval from the London School of Hygiene \& Tropical Medicine (\#11976), the Ethics Review Board of MSF (\#1708) and the Commission Institutionnelle d'Ethique of the Catholic University of Bukavu (UCB/ClE/NC/005B/2017). Informed consent was obtained from all participants or their carers prior to participation. Young adolescents received information appropriate for their age before they were asked for consent.

Study participants were passively recruited at MSF clinics from pregnant women presenting for ANC and from patients presenting with signs/symptoms of malaria (axillary temperature $\geq 37.5{ }^{\circ} \mathrm{C}$ or history of fever). Malaria HRP2-test rapid diagnostic tests (RDTs) (SD BIOLINE Malaria Ag P.f, 05FK50; Gyeonggi-do, Republic of Korea) were performed to detect presence of $P$. falciparum antigen. Two blood spots were collected on filter papers (Whatman ${ }^{\circledR} 903$ Protein Saver Card; Maidstone, UK), air-dried, stored individually in zip-lock bags with desiccant at room temperature, and kept away from humidity, excessive heat and light. All dried blood spots (DBS) samples, whether RDT positive or negative, were shipped at ambient temperature to Radboud University Medical Centre, The Netherlands, for further processing.

\section{Laboratory procedures}

DNA was extracted from two 3-mm DBS punches using Chelex ${ }^{\circledR} 100$ chelatin resin, according to the manufacturer's recommendations, and using a filter (WHA77002808 ALDRICH Whatman ${ }^{\circledR}$ UNIFILTER $^{\circledR}$ plates) to avoid Chelex in the final DNA sample. DNA from both RDT positive and negative samples was tested using a $\mathrm{qPCR}$ assay targeting the P. falciparum-specific $18 \mathrm{~S}$ gene [15]. This assay was originally optimized for use on whole blood samples (and not filter paper samples). For the current project, parasite density was quantified using in vitro trendline material on filter paper (Whatman ${ }^{\circledR}$ 903 Protein Saver Card; Maidstone, UK) to have the same conditions for test samples and trendlines. For all qPCR positive samples, nested PCR amplification was done for dhps; for a sub-set of samples where dhps amplification was successful, $d$ fhr was also amplified $(\mathrm{n}=387)$. Primers were based on previous publications $[16,17]$ and are presented in Table 1. PCR conditions were $94{ }^{\circ} \mathrm{C} \times 3 \mathrm{~min}$, followed by 40 rounds of $94{ }^{\circ} \mathrm{C} \times 1 \mathrm{~min} \rightarrow 51{ }^{\circ} \mathrm{C} \times 2 \mathrm{~min}$ $\rightarrow 72{ }^{\circ} \mathrm{C} \times 1 \mathrm{~min}$, and a final round of $72{ }^{\circ} \mathrm{C}$ for $10 \mathrm{~min}$. Samples were evaluated by gel electrophoresis prior to further analysis.

dhps K450E and A581G and dhfr I164L were assessed by nested PCR followed by allele specific primer extension (ASPE) and detection on a Luminex instrument (MAGPIX $^{\circledR}$; Luminex Corp) with xPONENT $^{\circledR} 4.2$ software. Briefly, amplified DNA was incubated with ExoSAPit for $30 \mathrm{~min}$ at $37{ }^{\circ} \mathrm{C}$, followed by $15 \mathrm{~min}$ at $80{ }^{\circ} \mathrm{C}$. Samples were then assembled with the ASPE master mix [250 nM each of ASPE probes and $200 \mu \mathrm{M}$ Biotin-14-dCTP (Invitrogen, UK)] using primers from Table 1 and incubated 30 cycles $\left(30 \mathrm{~s} 90{ }^{\circ} \mathrm{C}, 1 \mathrm{~min}\right.$ $\left.53.5{ }^{\circ} \mathrm{C}, 2 \min 72{ }^{\circ} \mathrm{C}\right)$. Utilizing different TAGs on different ASPE primers, multiple magnetic bead sets could be hybridized to the ASPE reactions. For each reaction, $1 \mu \mathrm{L}$ of each bead set was used. After two washes with Tm buffer $(0.2 \mathrm{M} \mathrm{NaCl}, 0.1 \mathrm{M}$ Tris, $0.08 \%$ TritonX-100, $\mathrm{pH} 8$ ), Tm buffer $+0.1 \%$ BSA $+2.5 \mu \mathrm{g} / \mathrm{mL}$ 
Table 1 Primer sequences

\begin{tabular}{|c|c|c|}
\hline Gene & Primer/probe & Sequence \\
\hline Pf18S & 185 forward & 5'GTAATTGGAATGATAGGAATTTACAAGGT 3' \\
\hline Pf18S & 185 reverse & 5'TCAACTACGAACGTTTTAACTGCAAC 3' \\
\hline Pf18S & 185 probe & 6FAM-AACAATTGGAGGGCAAG-MGBNFQ \\
\hline Dhps & Outer forward & 5' GATTCTTTTTCAGATGGAGG 3' \\
\hline Dhps & Outer reverse & $5^{\prime}$ TTCCTCATGTAATTCATCTGA $3^{\prime}$ \\
\hline Dhps & Inner forward & 5' AACCTAAACGTGCTGTTCAA 3' \\
\hline Dhps & Inner reverse & 5' AATTGTGTGATTTGTCCACAA $3^{\prime}$ \\
\hline Dhfr & Outer forward & $5^{\prime}$ TGATGGAACAAGTCTGC $3^{\prime}$ \\
\hline Dhfr & Outer reverse & 5' ACTTTGTTTATTTCCATTCA 3' \\
\hline Dhfr & Inner forward & 5'TGTGCATGTTGTAAGGTTGA 3' \\
\hline Dhfr & Inner reverse & 5' GATACTCATTTTCATTTATTTCTGGA 3' \\
\hline Dhps & $540 \mathrm{~K}$ & 5' ATTAAACAACTCTTAACTACACAAGGAAATCCACATACAATGGATA 3' \\
\hline Dhps & $540 \mathrm{E}$ & 5' CTTTCTTAATACATTACAACATACGAAATCCACATACAATGGATG 3' \\
\hline Dhps & $581 \mathrm{~A}$ & 5' CATAAATCTTCTCATTCTAACAAATTGATATTGGATTAGGATTTGC $3^{\prime}$ \\
\hline Dhps & $581 \mathrm{G}$ & 5' CAAACAAACATTCAAATATCAATCTTGATATTGGATTAGGATTTGG 3' \\
\hline Dhfr & $164 \mid$ & 5' CATAAATCTTCTCATTCTAACAAAGAAATTAAATTACTATAAATGTTTTATTA $3^{\prime}$ \\
\hline Dhfr & $164 \mathrm{~L}$ & 5' CAAACAAACATTCAAATATCAATCGAAATTAAATTACTATAAATGTTTTATTT 3' \\
\hline
\end{tabular}

Listed are primer sequences used in qPCR to confirm $P$. falciparum infection and quantify parasite density (Pf18S), nested PCR for amplifying Dhps and Dhfr for ASPE reactions for hybridisation with different alleles. Sequence highlighted in italic is the Luminex tag sequence

$q P C R$ quantitative polymerase chain reaction, Dhps dihydropteroate synthase; Dhfr dihydrofolate reductase, ASPE Allele Specific Primer Extension

Streptavidin-R-phycoerythrin (SAPE) was added to each sample. Samples were analysed at $37^{\circ} \mathrm{C}$ on the Luminex analyser according to manufacturer's instructions.

\section{Statistical analysis}

Statistical analyses were performed in STATA version 15.0 (StataCorp; College Station, TX, USA). Data were typically presented per site and separately for sample donors recruited at clinics with suspected malaria (suspected clinical cases) or pregnant women attending antenatal care (ANC participants). Age was categorized in 5 categories (<2 years, 2-4 years, 5-9 years, 10-14 years, $\geq 15$ years). Parasite density estimates were compared between RDT-positive and -negative individuals by nonparametric Wilcoxon rank-sum test; median values with 25th and 75th percentile (IQR) were presented. Parasite prevalence estimates were given for suspected clinical cases and ANC participants separately, adjusting 95\% CI for the cluster-design of data collection using the survey (svy) command in STATA. Genotyping results were presented per site and separately for suspected clinical cases and ANC participants.

\section{Results}

RDT and qPCR results were available for a total of 2421 individuals from the four study sites; data were unavailable for 83 additional participants because of missing blood spot samples $(n=8)$, errors in identification codes $(n=3)$ and laboratory errors/assay failures $(n=72)$. The majority of participants with successfully analysed samples were collected at clinics from suspected clinical malaria cases $(78.8 \%, 1907 / 2421)$, the remainder coming from ANC participants (Table 2). Across study sites, parasite prevalence by RDT was higher in suspected malaria cases $(45.4 \%, 95 \%$ CI 31.7-59.9) compared to ANC participants $(23.2 ; 95 \%$ CI $6.8-55.6 \%)(\mathrm{p}=0.026)$, taking into account a study site as primary sampling unit. Similarly, parasite prevalence by qPCR was higher in suspected malaria cases $(78.0 \%, 95 \%$ CI 72.1-82.9) compared to ANC participants (48.4; 95\% CI 25.9-71.6\%) $(\mathrm{p}=0.029)$. Individuals who were RDT-positive were significantly more likely to be qPCR-positive compared to RDT-negative individuals (OR 13.8, 95\% CI 3.49-54.4, $\mathrm{p}=0.009$ ). Nevertheless, $47.8-67.4 \%$ of RDT-negative suspected clinical cases and 25.0-52.0\% of RDT-negative ANC participants were found to be qPCR-positive in the four study sites. The estimated median parasite density by qPCR in RDT-positive individuals was 13,608 parasites/ $\mu \mathrm{L}$ (IQR 384-108,600) compared to 2.3 parasites/ $\mu \mathrm{L}$ (IQR 0.5-25.2) in RDT-negative individuals (Fig. 2; $\mathrm{p}<0.001)$.

Amplification of the dhps gene was successful for $86.2 \%$ (937/1086) of RDT-positive samples and 55.5\% (361/651) of RDT-negative/qPCR-positive samples. All dhps positive samples were genotyped for dhps K540E and A581G and categorized as having a pure or mixed genotype for 
Table 2 Characteristics of study participants

\begin{tabular}{|c|c|c|c|c|}
\hline & Baraka & Kimbi & Walikale & Mweso \\
\hline Suspected clinical malaria & 500 & 415 & 662 & 330 \\
\hline Antenatal visits & 169 & 151 & 73 & 121 \\
\hline \multicolumn{5}{|l|}{ Age, $\%(n)$} \\
\hline$<2$ years & $19.9(133)$ & $21.8(123)$ & $14.3(105)$ & $12.0(54)$ \\
\hline $2-4$ years & $20.9(140)$ & $30.3(171)$ & $17.1(125)$ & $13.1(59)$ \\
\hline $5-9$ years & $17.2(115)$ & $2.1(12)$ & $17.9(131)$ & $13.3(60)$ \\
\hline $10-14$ years & $6.4(43)$ & $1.6(9)$ & $14.6(107)$ & $11.3(51)$ \\
\hline$\geq 15$ years & $35.6(238)$ & $44.2(249)$ & $36.2(265)$ & $50.3(227)$ \\
\hline \multicolumn{5}{|c|}{ Parasite prevalence RDT, \% (n/N) } \\
\hline Clinic & $47.2(236 / 500)$ & $50.6(210 / 415)$ & $64.1(424 / 662)$ & $51.8(171 / 330)$ \\
\hline ANC & $9.5(16 / 169)$ & $32.5(49 / 151)$ & $50.7(37 / 73)$ & $14.1(17 / 121)$ \\
\hline \multicolumn{5}{|c|}{ Parasite prevalence $\mathrm{qPCR}, \%(\mathrm{n} / \mathrm{N})$} \\
\hline Clinic & $81.2(406 / 500)$ & $74.9(311 / 415)$ & $79.8(528 / 662)$ & $73.3(242 / 330)$ \\
\hline ANC & $41.4(70 / 169)$ & $64.9(98 / 151)$ & $57.5(42 / 73)$ & $32.2(39 / 121)$ \\
\hline
\end{tabular}

Clinic samples from individuals attending the clinic with suspected clinical malaria, $A N C$ samples from individuals attending antenatal care, $R D T$ rapid diagnostic test SD Bioline, $q P C R$ quantitative polymerase chain reaction, $n$ absolute number, $N$ total sample size per location

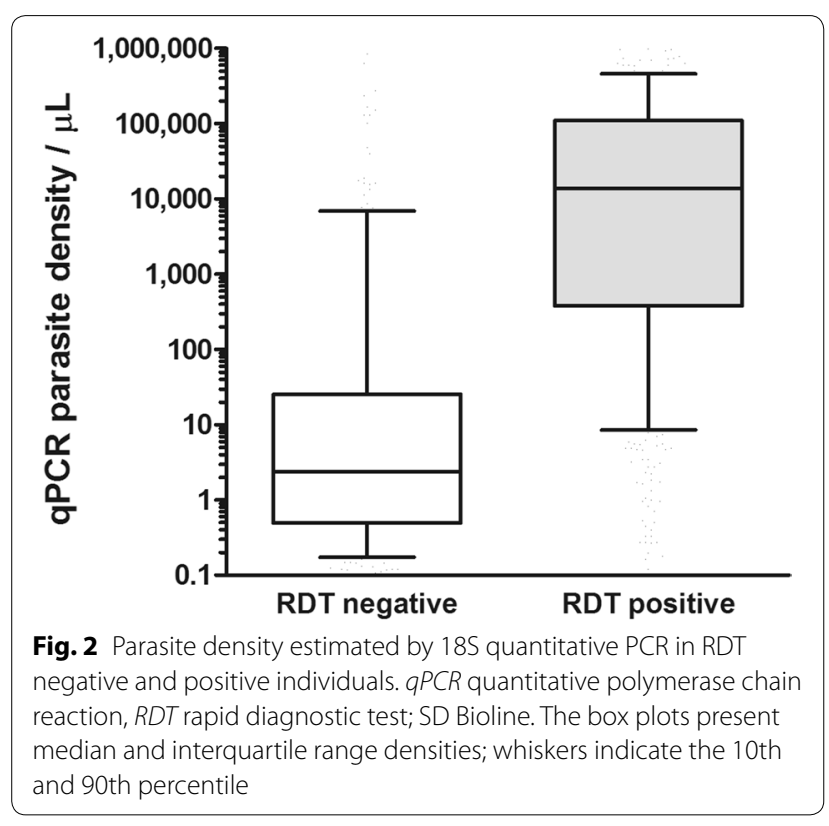

each locus (Table 3, Additional file 1: Table S1). The presence of dhps K540E, either as pure mutant or as mixed mutant/wild-type infections, exceeded $50 \%$ in all study sites, with strong evidence for differences between sites ( $<<0.001$ ) (Fig. 3). dhps A581G mutants were less prevalent than dhps K540E; only in Mweso approximately half of all infections carried the $581 \mathrm{G}$ as pure mutant or mixed wild-type/mutant. Again, there was strong evidence for differences in the prevalence of the dhps A581G mutation between sites $(\mathrm{p}<0.001)$. The dhps
A581G mutation almost exclusively occurred in the presence of the dhps K540E mutation: $100.0 \%$ (143/143) of all dhps A581G pure mutant and 96.2 (128/133) of all mixed wildtype/mutant infections had concurrent mutations in dhps K540E.

The $d h f r$ gene was successfully amplified in $94.9 \%$ $(333 / 351)$ of randomly selected samples (Baraka: $\mathrm{n}=78$; Kimbi: $\mathrm{n}=91$; Walikale: $\mathrm{n}=96$; Mweso: $\mathrm{n}=68$ ). Among these samples, the $d h f r$ I164L mutation was found in only one sample $(0.3 \%, 1 / 333)$. The sample was collected from an RDT-confirmed clinical malaria case from Walikale and was wild type for dhps K540E and A581G.

\section{Discussion}

High numbers of malaria cases continue to pose an intense burden to DRC and to the individuals affected. Due to conflict and unrest in the country, there is limited current information available on parasite resistance to anti-malarial drugs. This knowledge gap hinders the informed use of anti-malarial interventions. The current study aimed to provide estimates of molecular markers conferring SP resistance in four settings in an area of chronic conflict in Eastern DRC.

Parasite prevalence among suspected malaria cases was uniformly high in the study settings. When sensitive qPCR was used to detect $P$. falciparum parasites, parasite prevalence was $70-80 \%$ among suspected malaria cases and $32-65 \%$ among women attending ANC visits. These findings confirm the high malaria burden in this part of DRC [18]. The higher prevalence of parasites by qPCR compared to RDT corroborates an increasing body of literature on low-density infections that are present in 
Table 3 The prevalence of mutations in Dhps 540 and Dhps 581 in the different study sites

\begin{tabular}{|c|c|c|c|c|}
\hline & Baraka & Kimbi & Walikale & Mweso \\
\hline & Clinic & Clinic & Clinic & Clinic \\
\hline $\mathrm{N}$ & 302 & 352 & 365 & 174 \\
\hline \multicolumn{5}{|l|}{ Dhps 540, \% (n) } \\
\hline Wildtype & $29.8(90)$ & $49.7(175)$ & $21.1(77)$ & $12.1(21)$ \\
\hline Pure mutant & $56.6(171)$ & $25.0(88)$ & $61.9(226)$ & $75.3(131)$ \\
\hline Mixed & $13.6(41)$ & $25.3(89)$ & $17.0(62)$ & $12.6(22)$ \\
\hline$N$ & 314 & 360 & 377 & 178 \\
\hline \multicolumn{5}{|l|}{ Dhps $581, \%(n)$} \\
\hline Wildtype & $78.0(245)$ & $87.2(314)$ & $78.8(297)$ & $52.8(94)$ \\
\hline Pure mutant & $12.1(38)$ & $4.4(16)$ & $10.1(38)$ & $30.3(54)$ \\
\hline Mixed & $9.9(31)$ & $8.3(30)$ & $11.1(42)$ & $16.9(30)$ \\
\hline
\end{tabular}

$N$ total sample size per location, $n$ absolute number, Dhps dihydropteroate synthase

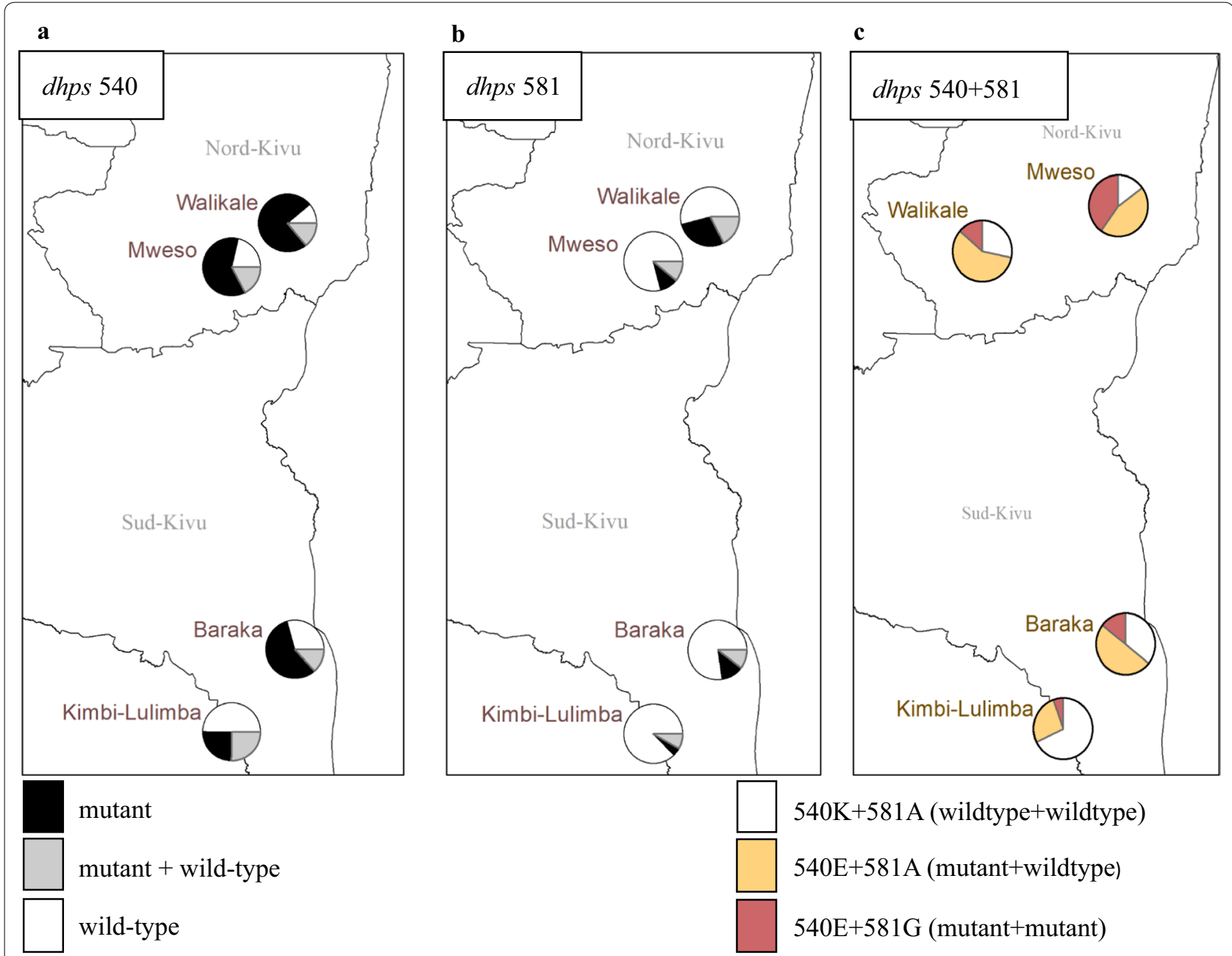

Fig. 3 Prevalence of dhps K540E and 581G mutations at the 4 MSF sites. The number of infections carrying pure mutant, mixed (mutant + wildtype) or pure wildtype infections are shown for dhps K540E (a), A581G (b) m and the haplotypes of dhps 540 and 581 at each site (c). dhps dihydropteroate synthase 
all age groups and across all endemicities in Africa and elsewhere [19, 20]. Parasite densities among RDT-negative qPCR-positive individuals were very low on average, below the threshold density typically detected by microscopy or RDT [19]. Whilst this suggests adequate performance of RDTs in the study settings in symptomatic cases, deletions in histidine-rich protein (HRP2), as described in DRC by Parr et al. [21] will be examined in the current dataset in future studies. It is currently unclear whether parasite densities below the threshold for detection by RDT contribute to acute clinical disease in semi-immune individuals, although low-density infections have been associated with haemolysis, inflammation, co-infection with invasive bacterial disease, cognitive impairment [22], and ongoing transmission of parasites to mosquitoes [23]. At present, it may be assumed that RDT-negative individuals presenting with suspected malaria had fever due to causes other than $P$. falciparum infection [24].

A high level of the dhps K540E mutation was observed in this region and this is consistent with studies that reviewed and mapped all publicly available data on $540 \mathrm{E}$ in Africa [9, 25, 26]. At all sites, the prevalence of dhps $K 540 E$ mutant exceeds $50 \%$, the threshold level defined by WHO to determine whether or not to introduce IPTi [27]. The highest prevalence of 540E was in the eastern sites (Mweso and Baraka), closest to the border with southwest Uganda and western Rwanda, a region previously identified as a hotspot with a high level of SP resistance $[25,26]$. This is consistent with the east-west gradient in the spatial distribution of the dhps mutations across DRC observed by Aydemir et al. [28] and summarized in Fig. 1. Okell et al. showed that resistance patterns are similar for sites up to $300 \mathrm{~km}$ apart [9] and Mweso is $<300 \mathrm{~km}$ from resistant hotspots in southwest Uganda $[29,30]$.

This study is consistent with a sequential build-up of resistance markers for SP. The A581G mutation almost exclusively occurred in the presence of K540E mutations [31]. The prevalence of I164L mutation on the dhfr gene was still very low, detected in only one of 333 samples. Future monitoring is needed to examine if the prevalence of this mutation is increasing and will have implications for SP efficacy. A relevant limitation of the current study is that samples were collected on one time-point only and solely collected at clinical facilities in suspected malaria cases and women attending ANC (routine screening of all pregnant women, including asymptomatically infected individuals). Some of the suspected malaria cases with measured fever and qPCR-or RDT-detected infections may have had fever due to causes other than malaria [24] and may have inappropriately received the diagnosis of clinical malaria. Whilst this misclassification is of no concern for the current objectives, estimating the prevalence of resistance markers among health facility attendees, it complicates a direct comparison of resistance in clinical versus asymptomatic infections.

The findings in this study are disappointing from a programmatic perspective. The study was initiated with the hope that IPTi could be introduced in the region to reduce morbidity and mortality. However, with resistance markers well above the threshold of 50\% (for K540E) this cannot be supported. Although WHO still recommends IPTp with SP in areas with high level of resistance to SP, based on studies in Tanzania and Malawi [32], the current findings of high prevalence of SP resistance among the target population suggest that the efficacy of IPTp in this setting should continue to be monitored [33] and alternative drugs such as dihydroartemisinin-piperaquine, mefloquine or doxycycline may be worth considering [34-36].

The current findings highlight significant challenges for the use of SP for chemoprevention in this region in DRC. It is therefore of utmost importance that other interventions, including vector control and prompt access to diagnosis and treatment, are implemented to the highest attainable standards to reduce exposure and prevent severe disease.

\section{Conclusion}

In the study areas of intense malaria transmission in North and South Kivu, the prevalence of the SP resistance marker dhps $\mathrm{K} 540 \mathrm{E}$ was above $50 \%$ in all study sites. K540E mutations regularly coincided with mutations in dhps A581G but not with the $d h f r$ I164L mutation. The current results do not support implementation of IPTi. Given the high morbidity and mortality from malaria in DRC, alternative approaches are needed for prevention and early treatment of malaria.

\section{Supplementary information}

Supplementary information accompanies this paper at https://doi. org/10.1186/s12936-019-3057-7.

Additional file 1: Table S1. The prevalence of mutations in Dhps 540 and Dhps 581 for sample donors with suspected clinical malaria and donors attending antenatal care.

\section{Abbreviations}

ANC: antenatal care; ASPE: allele specific primer extension; DBS: dried blood spots; dhfr: dihydrofolate reductase; dhps: dihydropteroate synthase; DNA: deoxyribonucleic acid; DRC: Democratic Republic of Congo; HRP2: histidinerich protein 2; ICCM: Integrated Community Case Management; IPTi-SP: intermittent preventive therapy for infants; IPTp: intermittent preventive treatment of malaria in pregnancy; IQR: interquartile range; RDT: malaria HRP2-test rapid diagnostic test; MSF: Médecins sans Frontières; MSF-OCA: Médecins sans Frontières-Operational Centre Amsterdam; OPD: outpatient department; qPCR: quantitative polymerase chain reaction; SMC: seasonal malaria chemoprophylaxis; SP: sulfadoxine-pyrimethamine; WHO: World Health Organization. 


\section{Acknowledgements}

Thanks to all laboratory technicians who collected the samples for their dedicated work: Gabriel Mulangano (Mweso), Gédéon Mulanda Monga (Kimbi), Justin Bazibuhe Bahati (Walikale), Pascal Kahindo Kabengwa (Baraka).

\section{Authors' contributions}

MvL designed and lead the work and drafted the manuscript. RvdM performed all lab analysis and drafted the manuscript. ML instructed all field lab staff and supervised collection of samples. She passed away in 2019 before submission of this manuscript. AO contributed to local design of the study, obtained ethical approval in DRC and supervised field staff in North Kivu. EB contributed to local design of the study, obtained ethical approval in DRC and supervised field staff In South Kivu. CB contributed to local design of the study, obtained ethical approval in DRC and supervised field staff in South Kivu. DC facilitated the design of the study to make it locally appropriate. LO contributed to the design of the study. EP was responsible for the field lab design of the study. LG was responsible for supervision of laboratory assays for resistance genotyping. KL was responsible for supervision of laboratory assays for DNA extraction and parasite quantification. VB Rao contributed to the design the study and wrote the study protocol. TB designed and supervised the study, the analysis and the writing of the manuscript. CR designed and supervised the study, the lab-analysis and the writing of the manuscript. All authors read and approved the final manuscript.

\section{Funding}

The funding for this study came from MSF-OCA.

\section{Availability of data and materials}

Data are available on request in accordance with MSF's data sharing policy at data.sharing@msf.org.

\section{Ethics approval and consent to participate}

The study received ethical approval from the London School of Hygiene \& Tropical Medicine (\#11976), the Ethics Review Board of Médecins Sans Frontières (\#1708) and the Commission Institutionnelle d'Ethique of the Catholic University of Bukavu (UCB/CIE/NC/005B/2017).

\section{Consent for publication}

Not applicable.

\section{Competing interests}

The authors declare that they have no competing interests.

\author{
Author details \\ ${ }^{1}$ Médecins Sans Frontières (MSF), Amsterdam, The Netherlands. ${ }^{2}$ Radboud \\ University Medical Centre, Nijmegen, The Netherlands. ${ }^{3}$ London School \\ of Hygiene and Tropical Medicine, London, UK. ${ }^{4}$ MSF, London, UK. ${ }^{5}$ Pro- \\ gramme National de Lutte contre le Paludisme (PNLP) South Kivu, Bukavu, \\ Democratic Republic of Congo. ${ }^{6}$ MSF, North Kivu, Goma, Democratic Republic \\ of Congo. ${ }^{7}$ MSF, Bukavu, Democratic Republic of Congo. ${ }^{8}$ Imperial College, \\ London, UK.
}

Received: 26 July 2019 Accepted: 7 December 2019 Published online: 18 December 2019

\section{References}

1. WHO. Country profile DRC malaria. Geneva: World Health Organization; 2018.

2. Doctor SM, Liu Y, Anderson OG, Whitesell AN, Mwandagalirwa MK, Muwonga J, et al. Low prevalence of Plasmodium malariae and Plasmodium ovale mono-infections among children in the Democratic Republic of the Congo: a population-based, cross-sectional study. Malar J. 2016;15:350.

3. Kavunga-Membo H, Ilombe G, Masumu J, Matangila J, Imponge J, Man-

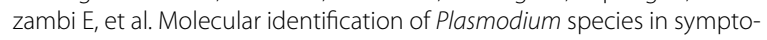
matic children of Democratic Republic of Congo. Malar J. 2018;17:334

4. Walker PGT, Floyd J, Kuile F, Cairns M. Estimated impact on birth weight of scaling up intermittent preventive treatment of malaria in pregnancy given sulphadoxine-pyrimethamine resistance in Africa: a mathematical model. PLoS Med. 2017;14:e1002243.

5. Maiga H, Lasry E, Diarra M, Sagara I, Bamadio A, Traore A, et al. Seasonal malaria chemoprevention with sulphadoxine-pyrimethamine and amodiaquine selects Pfdhfr-dhps quintuple mutant genotype in Mali. PLoS ONE. 2016;11:e162718.

6. Grais RF, Laminou IM, Messe LW, Makarimi R, Bouriema SH, Langendorf C, et al. Molecular markers of resistance to amodiaquine plus sulfadoxine-pyrimethamine in an area with seasonal malaria chemoprevention in south central Niger. Malar J. 2018;17:98.

7. Kublin JG, Dzinjalamala FK, Kamwendo DD, Malkin EM, Cortese JF, Martino LM, et al. Molecular markers for failure of sulfadoxine-pyrimethamine and chlorproguanil-dapsone treatment of Plasmodium falciparum malaria. J Infect Dis. 2002;185:380-8.

8. Staedke SG, Sendagire H, Lamola S, Kamya MR, Dorsey G, Rosenthal PJ. Relationship between age, molecular markers, and response to sulphadoxine-pyrimethamine treatment in Kampala, Uganda. Trop Med Int Health. 2004;9:624-9.

9. Okell LC, Griffin JT, Roper C. Mapping sulphadoxine-pyrimethamineresistant Plasmodium falciparum malaria in infected humans and in parasite populations in Africa. Sci Rep. 2017;7:7389.

10. WHO. Policy recommendation on Intermittent Preventive Treatment during infancy for Plasmodium falciparum malaria control in Africa. Contraindications. Geneva: World Health Organization; 2009.

11. Chico RM, Cano J, Ariti C, Collier TJ, Chandramohan D, Roper C, et al. Influence of malaria transmission intensity and the $581 \mathrm{G}$ mutation on the efficacy of intermittent preventive treatment in pregnancy: systematic review and meta-analysis. Trop Med Int Health. 2015;20:1621-33.

12. Desai M, Gutman J, Taylor SM, Wiegand RE, Khairallah C, Kayentao K, et al. Impact of sulfadoxine-pyrimethamine resistance on effectiveness of intermittent preventive therapy for malaria in pregnancy at clearing infections and preventing low birth weight. Clin Infect Dis. 2016:62:323-33.

13. Kayentao K, Garner P, Van Eijk AM, Naidoo I, Roper C, Mulokozi A, et al. Intermittent preventive therapy for malaria during pregnancy using 2 vs 3 or more doses of sulfadoxine-pyrimethamine and risk of low birth weight in Africa: systematic review and meta-analysis. JAMA. 2013;309:594-604.

14. WHO. Evidence Review Group: Intermittent Preventive Treatment of malaria in pregnancy (IPTp) with sulfadoxine-pyrimethamine (SP). Geneva: World Health Organization; 2017. p. 1-17.

15. Hermsen CC, Telgt DS, Linders EH, van de Locht LA, Eling WM, Mensink EJ, et al. Detection of Plasmodium falciparum malaria parasites in vivo by real-time quantitative PCR. Mol Biochem Parasitol. 2001;118:247-51.

16. Pearce RJ, Drakeley C, Chandramohan D, Mosha F, Roper C. Molecular determination of point mutation haplotypes in the dihydrofolate reductase and dihydropteroate synthase of Plasmodium falciparum in three districts of Northern Tanzania. Antimicrob Agents Chemother. 2003:47:1347-54

17. Barnadas C, Timinao L, Javati S, Iga J, Malau E, Koepfli C, et al. Significant geographical differences in prevalence of mutations associated with Plasmodium falciparum and Plasmodium vivax drug resistance in two regions from Papua New Guinea. Malar J. 2015;14:399.

18. Gething PW, Casey DC, Weiss DJ, Bisanzio D, Bhatt S, Cameron E, et al. Mapping Plasmodium falciparum mortality in Africa between 1990 and 2015. N Engl J Med. 2016;375:2435-45

19. Slater HC, Ross A, Felger I, Hofmann NE, Robinson L, Cook J, et al. The temporal dynamics and infectiousness of subpatent Plasmodium falciparum infections in relation to parasite density. Nat Commun. 2019;10:1433.

20. Hofmann NE, Gruenberg M, Nate E, Ura A, Rodriguez-Rodriguez D, Salib $M$, et al. Assessment of ultra-sensitive malaria diagnosis versus standard molecular diagnostics for malaria elimination: an in-depth molecular community cross-sectional study. Lancet Infect Dis. 2018;18:1108-16.

21. Parr JB, Verity R, Doctor SM, Janko M, Carey-Ewend K, Turman BJ, et al. Pfhrp2-deleted Plasmodium falciparum parasites in the Democratic Republic of the Congo: a national cross-sectional survey. J Infect Dis. 2017;216:36-44.

22. Chen I, Clarke SE, Gosling R, Hamainza B, Killeen G. "Asymptomatic" malaria: a chronic and debilitating infection that should be treated. PLos Med. 2016;13:e1001942. 
23. Goncalves BP, Kapulu MC, Sawa P, Guelbeogo WM, Tiono AB, Grignard $L$, et al. Examining the human infectious reservoir for Plasmodium falciparum malaria in areas of differing transmission intensity. Nat Commun. 2017;8:1133.

24. D'Acremont V, Kilowoko M, Kyungu E, Philipina S, Sangu W, Kahama-Maro $J$, et al. Beyond malaria-causes of fever in outpatient Tanzanian children. N Engl J Med. 2014;370:809-17.

25. Naidoo I, Roper C. Drug resistance maps to guide intermittent preventive treatment of malaria in African infants. Parasitology. 2011;138:1469-79.

26. Naidoo I, Roper C. Mapping 'partially resistant', 'fully resistant', and 'super resistant' malaria. Trends Parasitol. 2013;29:505-15.

27. WHO. Guidelines for the treatment of malaria. 2nd ed. Geneva: World Health Organization; 2010. p. 197.

28. Aydemir O, Janko M, Hathaway NJ, Verity R, Mwandagalirwa MK, Tshefu AK, et al. Drug-resistance and population structure of Plasmodium falciparum across the Democratic Republic of Congo using high-throughput molecular inversion probes. J Infect Dis. 2018;218:946-55

29. Lynch C, Pearce R, Pota H, Cox J, Abeku TA, Rwakimari J, et al. Emergence of a dhfr mutation conferring high-level drug resistance in Plasmodium falciparum populations from southwest Uganda. J Infect Dis. 2008;197:1598-604.

30. Lynch CA, Pearce R, Pota H, Egwang C, Egwang T, Bhasin A, et al. Travel and the emergence of high-level drug resistance in Plasmodium falciparum in southwest Uganda: results from a population-based study. Malar J. 2017:16:150.
31. Alifrangis $M$, Nag S, Schousboe ML, Ishengoma D, Lusingu J, Pota H, et al. Independent origin of Plasmodium falciparum antifolate super-resistance, Uganda, Tanzania, and Ethiopia. Emerg Infect Dis. 2014;20:1280-6.

32. WHO. Policy brief for the implementation of intermittent preventive treatment of malaria in pregnancy. April 2013 (revised Jan 2014). Geneva: World Health Organization; 2013. p. 2014.

33. WHO. Evidence review group on intermittent preventive treatment (IPT of malaria in pregnancy). Geneva: World Health Organization; 2013. p. $1-17$.

34. Jagannathan P, Kakuru A, Okiring J, Muhindo MK, Natureeba P, Nakalembe $M$, et al. Dihydroartemisinin-piperaquine for intermittent preventive treatment of malaria during pregnancy and risk of malaria in early childhood: a randomized controlled trial. PLoS Med. 2018;15:e1002606.

35. Gosling RD, Gesase S, Mosha JF, Carneiro I, Hashim R, Lemnge M, et al. Protective efficacy and safety of three antimalarial regimens for intermittent preventive treatment for malaria in infants: a randomised, doubleblind, placebo-controlled trial. Lancet. 2009;374:1521-32.

36. González R, Piqueras M, Jj A, Fo K, Menéndez C. Mefloquine for preventing malaria in pregnant women. Cochrane Database Syst Rev. 2018;11:CD011444.

\section{Publisher's Note}

Springer Nature remains neutral with regard to jurisdictional claims in published maps and institutional affiliations.
Ready to submit your research? Choose BMC and benefit from:

- fast, convenient online submission

- thorough peer review by experienced researchers in your field

- rapid publication on acceptance

- support for research data, including large and complex data types

- gold Open Access which fosters wider collaboration and increased citations

- maximum visibility for your research: over $100 \mathrm{M}$ website views per year

At BMC, research is always in progress.

Learn more biomedcentral.com/submissions 